\title{
Multidisciplinary Approach to Older Adults with Hematologic Malignancies—a Paradigm Shift
}

\author{
Sarah A. Wall ${ }^{2} \cdot$ Erin Stevens $^{1} \cdot$ Jennifer Vaughn ${ }^{2} \cdot$ Naresh Bumma $^{2} \cdot$ Ashley E. Rosko $^{2} \cdot$ Uma Borate $^{2}$
}

Accepted: 14 July 2021 / Published online: 13 January 2022

(c) The Author(s), under exclusive licence to Springer Science+Business Media, LLC, part of Springer Nature 2021

\begin{abstract}
Hematologic malignancies are most likely to present in the seventh and eighth decades of life. Continued population growth will lead to increasing numbers of older adults with hematologic malignancies. Oncology care for older adults is complex and must account for the effect of aging on disease biology and treatment tolerance. Multidisciplinary oncology care has been utilized in solid tumor oncology for decades, initially driven by the need for multi-modality treatment. In this review, we make the case for multidisciplinary oncogeriatric care for older adults with hematologic malignancies in order to best navigate the intersection of aging and blood cancer.
\end{abstract}

Keywords Multidisciplinary $\cdot$ Hematology $\cdot$ Geriatric Oncolog

Teamwork in health research and healthcare delivery is increasingly valued and emphasized, particularly in the fields of gerontology and oncology. The National Institute of Health began solicitation of multiple principal investigator grants in 2006 when only three such grants were funded and that number increased to 1098 funded grants by 2013 [1]. Growth of team science has led to use of terms such as multidisciplinary and interdisciplinary, often with ambiguous definition and interchangeable use. We have found the following definitions, as elucidated by Choi and Pak [2], to be helpful in distinguishing between the two. Multidisciplinarity draws on knowledge from different specialists working in series or parallel and each stays within the boundaries of their field. Interdisciplinarity includes different specialists working together to synthesize and harmonize links between their respective fields to create a coordinated, coherent plan. Based on these definitions, some "multidisciplinary" clinics actually function as interdisciplinary teams, collaborating on development of plan of care based on shared input from team members. In this review, we will highlight the roles for

This article is part of the Topical Collection on Health Economics

Sarah A. Wall

sarah.wall@osumc.edu

1 Division of Palliative Medicine, Columbus, USA

2 Division of Hematology, Ohio State University, Columbus, OH 43210, USA interdisciplinary and multidisciplinary collaboration in the care of older adults with hematologic malignancies.

\section{Geriatric Multidisciplinary Care}

A key feature to geriatric care is an approach to address the multidimensional needs of an aging or frail older adult. The health and social service needs overlap, creating complexity among the emotional, financial, social, and medical issues. A single health provider usually does not have the resources or expertise to manage these needs and an interdisciplinary approach has become the hallmark for geriatric care [3]. This specialized model involves providers with competency in geriatrics and care is provided and coordinated throughout the continuum of care. There is not a defined structure but the core interdisciplinary team often consists of a geriatrician, nurse practitioner or nurse, and social worker [3]. Some practice settings have an extended team that may include other professionals such as pharmacists, physical therapists, dieticians, and home-health nurses [4]. The providers work together in the same setting and perform separate assessments though domains may overlap. For example, aspects of a functional assessment may be addressed by both the physical therapist and the geriatrician. The team communicates recommendations at an interdisciplinary meeting, usually held at least once a week, to review complex care plans and achieve a shared plan for the patient $[2,5]$. 
While members of the interdisciplinary team may vary, the unifying approach of care to address the multidimensional geriatric needs is a comprehensive geriatric assessment (CGA) [6]. The CGA is a screening tool designed to better understand the health and needs of an aging adult. It assesses cognition, function, sensory, nutrition, medications, comorbid conditions, affect, and environmental and social supports [7]. Common Instruments used in the CGA include self-reported Instrumental Activities of Daily Living and Activities of Daily Living, the Timed Up and Go, the Geriatric Depression Scale, and the MiniCog or Clock Drawing Test [8]. Instruments selected may vary based on patient demographics, clinic structure, and interdisciplinary team preferences. The John A. Hartford Foundation has a list of tools with instructional videos listed on their website that can be used in the clinical setting [9]. The principles of geriatric management and the CGA are increasingly being applied to medical subspecialties. While geriatricians often use this information to focus on maintaining patient autonomy, reducing the risk of hospitalization, and improving overall support, other specialists may use this information to predict risk of treatment toxicity, postoperative mortality, or chances of survival with treatment [10].

\section{Malignant Hematology Multidisciplinary Care}

Due to the need for multiple consultations from medical, radiation, and surgical oncologists, multidisciplinary clinics have become common in the care of solid tumors [11-15]. However, creating multidisciplinary clinics in hematologic malignancies is an evolving concept that, depending on the disease of interest, may require an often broader set of expertise that extends to fields outside of oncology. Traditionally, treatment decisions in hematologic malignancies are made by the medical hematologist/oncologist. The rapidly evolving landscape of genetic and molecular testing in these diseases, however, has required the hematopathologist to take an active role in both diagnosis and prognostication for these patients [16-18]. In addition, as survival has increased from various hematologic diseases over the last few decades, the opportunity for patients to develop comorbidities as a direct result of the disease or treatment exposure has necessitated the incorporation of disciplines such as cardiology, nephrology, neurology, infectious disease, pulmonology, and dermatology, among others. Many hematologic cancers also require patients and providers to engage in shared decisionmaking regarding the selection of therapies. As patients with acute leukemias, myeloma, and lymphomas are often faced with the choice of receiving aggressive and often more toxic treatment regimens versus less-toxic palliative therapies, the inclusion of geriatric medicine and palliative care into upfront discussions of patient management has also shown promise $[19,20]$.

\section{Supporting Evidence for the Role of Multidisciplinary Care in Oncogeriatrics}

The role of multidisciplinary care in oncology clinics has grown over the last three decades, driven by the need for collaboration between medical, surgical, and radiation oncology subspecialists [21,22]. Early manifestations of multidisciplinary care often took the form of tumor board meetings rather than clinical encounters directly involving patients. More recently, multidisciplinary, "one-stop oncology care" clinics have led to increased patient satisfaction $[23,24]$ and decreased time to treatment [14, 25-27]. With more experience and refinement, many multidisciplinary oncology clinics have been able to demonstrate improved quality of life [28, 29] and increased appropriateness of treatment (i.e., curative versus palliative intent) [30, 31]. In addition, multidisciplinary programs have led to more consistent adherence to national guidelines for treatment and offer unique educational opportunities for trainees [14].

The supporting evidence for multidisciplinary clinics specializing in breast and lung cancer is most robust, likely because of the prevalence of these diagnoses and their frequent need for multi-modality treatment $[14,21,24$, $25,31]$. Some multidisciplinary oncology clinics not only focus on a specific cancer subtype, but also on the effect of increasing age on cancer biology and response to and tolerability of treatment. Disappointingly, a scoping review of multidisciplinary care for patients with colorectal cancer demonstrated that older age negatively influenced access to multidisciplinary care with older patients less likely to be discussed by a multidisciplinary team [32] Fortunately, oncogeriatric-specific multidisciplinary teams are increasingly utilized in evaluation of older adults with cancer. In a recent study, patients 70 years or older with solid tumor malignancy were reviewed at surgical oncology tumor board and in an oncogeriatric-specific multidisciplinary team after completing geriatric assessment [33]. The recommendation from the oncogeriatric multidisciplinary team led to treatment modification in $25 \%$ of patients with similar 1-year mortality between patients with modified versus unmodified treatment plan $(26.1 \%$ vs. $29.7 \%, p=0.7)$. The modified treatment plan resulted in less surgery and less treatment with curative intent but also fewer complications and less time spent in the hospital.

Most hematologic malignancies are treated primarily, often exclusively, with systemic chemo- or immunotherapy prescribed by a medical oncologist or hematologist. 
As such, management of hematologic malignancies has been historically underrepresented in traditional models of multidisciplinary oncology care. Multidisciplinary care in hematologic malignancies has been reported in a few different specialized settings, most commonly in evaluation for hematopoietic cell transplantation [34-36], but also in subspecialty clinics for cutaneous lymphoma [37] and clonal hematopoiesis [38].

An emerging interest in the care of patients with hematologic malignancies is the impact of advancing age on prognosis, treatment tolerability and efficacy, and quality of life. An increasing rate of population aging worldwide [39] paired with median age of onset for most hematologic malignancies in the seventh decade [40] will lead to significant growth in the number of older patients with hematologic malignancies who will require specialized care. It is increasingly well recognized that older adults with hematologic malignancies require a different approach than younger patients $[41,42]$. Guidance is available for treatment recommendations based on "fitness" or "candidacy for intensive treatment" though these terms are often not well defined $[42,43]$.

In a busy clinical oncology practice, brief geriatric assessment tools can be practical screening tools and may be incorporated into treatment planning. There are two diseasespecific tools developed for use in patients with multiple myeloma: the International Myeloma Working Group frailty score [44] and the revised Myeloma Comorbidity Index [45]. The first uses information readily available through chart review and patient interview while the latter requires measurement of grip strength and gait speed, and both are brief tools that have been validated as predictors of overall survival. A similar modified tool, the Elderly Prognostic Index, has also been recently validated for prediction of overall survival among older adults with diffuse large B-cell lymphoma [46]. More generalizable, the prognostic value of geriatric assessment in hematologic malignancies as a group has been demonstrated across a number of studies. In a systematic review of geriatric assessment in older adults with hematologic malignancy, 44 applicable studies were identified with 15 including multiple hematologic malignancy subtypes [47]. Overall, frailty (variably measured across studies), instrumental activities of daily living, nutritional status, and polypharmacy were all relevant in prediction of mortality, regimen-related toxicity, and non-completion of treatment.

Moving beyond prognostication, geriatric assessment should be utilized not only to identify geriatric syndromes or vulnerabilities, but also to address them [48]. As outlined by Goede and Stauder [19], incorporation of oncogeriatric principles in the hematology clinic through multidisciplinary care should include a routinely performed geriatric screening or assessment and access to multidisciplinary-driven geriatric intervention. Supporting evidence for the benefit of geriatric intervention is not yet widely available. Promising evidence for mortality benefit due to geriatric intervention comes from two studies of hematopoietic cell transplantation candidates $[35,36]$. Among patients $\geq 60$ years of age receiving allogeneic hematopoietic cell transplant, 1-year overall survival improved to $70 \%$ for patients evaluated and treated in a transplant optimization multidisciplinary clinic compared to only $43 \%$ in the historical control group [36]. This was driven in part by decrease in non-relapse mortality from 43 to $18 \%$. Among older autologous hematopoietic cell transplant recipients (median age 71 years), evaluated in the same transplant optimization multidisciplinary clinic, $68 \%$ of patients (62/91) proceeded with transplant with $20 \%$ of those (13/62) deferred after initial evaluation to allow time for optimization [35]. The 1-year overall survival was identical at $92 \%$ for both recipients proceeding directly to transplant and those who were initially deferred.

The benefit of geriatric intervention may be seen through traditional outcomes like mortality and regimen-related toxicity, though application of geriatric intervention can be tailored toward optimization of a number of outcomes, most importantly, patient-specific goals of care. There are currently 42 active studies at clinicaltrials.gov exploring nonpharmacologic interventions in older adult patients with

Table 1 Diagnostic, prognostic, and therapeutic implications of multidisciplinary oncogeriatric care

\begin{tabular}{|c|c|c|}
\hline Diagnosis & Prognosis & Therapeutics \\
\hline Identify: & Predict: & $\begin{array}{l}\text { Ancillary services (physical/occupational therapy, psy- } \\
\text { chotherapy, hearing aid evaluation) }\end{array}$ \\
\hline Frailty & Mortality & Self-administered (exercise or nutrition programs) \\
\hline Inappropriate medications/polypharmacy & Regimen-related toxicity & Physician specialist referral \\
\hline Sensory deficits & Response to therapy & De-prescription (or dose modification) \\
\hline Cognitive impairment & Quality of life & Support services (home healthcare, grants, transportation) \\
\hline Gaps in social support & Healthcare resource utilization & Treatment plan modification \\
\hline Financial toxicity & & Senolytic drugs \\
\hline
\end{tabular}


cancer and utilizing mortality, healthcare utilization, and quality of life-based endpoints. Of those 42 studies, nine are specific to hematologic malignancy diagnoses [49]. A summary of the diagnostic, prognostic, and therapeutic implications of multidisciplinary oncogeriatric care is provided in Table 1.

\section{Our Multidisciplinary Hematology and Oncogeriatric Clinics}

The Comprehensive Amyloidosis Clinic (CAC) at Ohio State University has been created to improve both the quality of care and outcomes of amyloidosis patients. The hematology team recognized the importance of early diagnosis of amyloidosis but desired a more collaborative and unified approach, which is accomplished through the development of this program, focusing on patient care, education, and research.

Amyloidosis is a complex disease that may affect many organs and often goes undiagnosed. As such, it is imperative to have a collaborative program with leadership in varied disciplines. Our CAC team is comprised of nephrologists, neurologists, cardiologists, hematologists, and physical therapists. The CAC runs biweekly with up to six patients scheduled on a half-day clinic. All patients arrive at the same time and providers rotate through individual patient rooms dependent on patient needs, especially for follow-up visits. The duration of the visit is typically $3 \mathrm{~h}$. An interdisciplinary team meeting occurs at the end of the clinic for treatment plan development. The strengths of the CAC include interdisciplinary collaboration in treatment planning, shared physical space to enhance efficiency for patients, experienced staff with expertise in amyloidosis and commitment to continuing education, and easy access to testing such as electrocardiography, urinalysis, echocardiography, remote telemetry, and electromyography.

The Cancer and Aging Resiliency (CARE) clinic is an interdisciplinary oncogeriatric clinic where we have been evaluating and treating older adults with hematologic malignancies since 2016. This interdisciplinary team includes a physical therapist, a dietitian, an audiologist, a clinical pharmacist, a social worker, a nurse case manager, a clinical nurse, and a physician. The physicians who staff the CARE clinic come from a variety of backgrounds including two with dual post-graduate training in gerontology and medical oncology, one with dual post-graduate training in palliative care and gerontology, and two with post-graduate hematology and medical oncology training who have pursued additional geriatric-specific education. At its inception, the CARE clinic was open to referrals of octogenarians with diagnosis of hematologic malignancies. Over time and with growth in the institutional Oncogeriatrics Program, the CARE clinic has expanded to include evaluations of any older adult with any cancer diagnosis. We have previously reported our experience in developing the CARE clinic and converting to a telehealth format during the COVID-19 pandemic [50, 51].

As a disease-agnostic clinic, CARE clinic focuses on identification of common geriatric syndromes and prescribed interventions to address them. General recommendations for oncology treatment plan modification may be made to the referring oncologist on the basis of the geriatric assessment, but are not prescribed by CARE physicians. The CARE clinic functions as a consultative resource to supplement the disease-specific care prescribed by our subspecialty experts. Similar to the CAC, CARE clinic runs over a halfday with up to six scheduled patients all arriving at the same time and visit duration of about $3 \mathrm{~h}$. CARE clinic meets every week. The strengths of the CARE clinic lie in the interdisciplinary approach to treatment plan development, shared physical space to enhance efficiency for patients, experienced staff with primary oncology training and commitment to continuing gerontology-specific education, and community outreach through educational programming for older adults with cancer diagnoses. A summary of the CAC and CARE clinic is provided in Table 2 for comparison of these two multidisciplinary approaches.

In addition to CAC and CARE clinic, we are in the process of developing a multidisciplinary clinic focusing on the diagnosis and treatment of myeloproliferative neoplasms (MPN). The primary goal of this clinic is to ensure more rapid and accurate diagnostic workup for MPN patients, as well as to ensure timely prognostication in order to expedite referral to high-risk clinics, clinical trials, and/ or allogeneic stem cell transplant if needed.

\section{Can We Move Management of Malignant Hematology Patients Earlier in the Disease Using a Multidisciplinary Approach?}

With the advent of NGS, the characterization of pre-malignant clonal hematopoiesis $(\mathrm{CH})$ in various forms such as CHIP and CCUS has been well described. These conditions, while increasing the risk of a subsequent myeloid neoplasm, also confer an increased risk of cardiovascular events. The importance of early recognition and management of these patients in a multidisciplinary setting including an experienced hematologist, a cardiologist, and a genetic counselor that can identify patients with co-occurring germline mutations and counsel family members appropriately has led to investment in the development of these clinics at several academic medical centers. At Ohio State University, we are developing an early detection high-risk clinic that will focus on Hematologic Abnormalities at risk of Leukemic Transformation (HALT). Our clinic will provide 
Table 2 Comparison of existing multidisciplinary oncology clinics at The James Cancer Hospital

\begin{tabular}{|c|c|c|}
\hline & Comprehensive amyloidosis clinic & Cancer and aging resiliency clinic \\
\hline Team members & $\begin{array}{l}\text { Hematologist } \\
\text { Cardiologist } \\
\text { Nephrologist } \\
\text { Neurologist } \\
\text { Physical therapist } \\
\text { Nurse case manager } \\
\text { Clinical nurse } \\
\text { Pharmacist }\end{array}$ & $\begin{array}{l}\text { Audiologist } \\
\text { Clinical nurse } \\
\text { Dietitian } \\
\text { Nurse case manager } \\
\text { Pharmacist } \\
\text { Physical therapist } \\
\text { Physician } \\
\text { Social worker }\end{array}$ \\
\hline Domains/specialties assessed & $\begin{array}{l}\text { Prescribing medications } \\
\text { Assessment for toxicity } \\
\text { Monitoring of therapy } \\
\text { Discussion for stem cell/cardiac transplant } \\
\text { Cardiac workup } \\
\text { Neurological assessment }\end{array}$ & $\begin{array}{l}\text { Sensory impairment } \\
\text { Cognition } \\
\text { Nutrition } \\
\text { Psychosocial support } \\
\text { Polypharmacy/medication management } \\
\text { Physical function } \\
\text { Goals of care }\end{array}$ \\
\hline Physical function assessment & $\begin{array}{l}\text { Short Physical Performance Battery } \\
\text { TUG } \\
\text { ADL/IADL }\end{array}$ & $\begin{array}{l}\text { Short Physical Performance Battery } \\
\text { TUG } \\
\text { ADL/IADL }\end{array}$ \\
\hline Clinic frequency and duration & $\begin{array}{l}\text { Biweekly half-day clinic } \\
6 \text { patients per clinic } \\
\text { 3-4-h visit }\end{array}$ & $\begin{array}{l}\text { Weekly half-day clinic } \\
6 \text { patients per clinic } \\
\text { 3-h visit }\end{array}$ \\
\hline Patient follow-up & Follow-up in clinic for patients depending on care & $\begin{array}{l}\text { No in-clinic follow-up } \\
\text { Nurse case manager coordinates refer- } \\
\text { rals for ongoing care needs }\end{array}$ \\
\hline
\end{tabular}

the multidisciplinary approach described above while also focusing on clinical and basic research in this emerging field.

\section{Why Development of Multidisciplinary Malignant Hematology Clinics Should Be the Norm}

The purpose of a multidisciplinary clinic is to provide patients with efficient, appropriate, and timely care for complex conditions. We aim to have a collaborative, multipronged approach in personalizing a therapeutic plan for complicated diseases while addressing challenges unique to the disease and specific to the health of the underlying individual. Different specialties participate in patient evaluation and care with the goal of optimization. Tertiary care centers often have the resources to combine basic and translational research in partnership with clinical application. Future investment is required in multi-facet programs for less common, but increasingly complex, hematologic malignancies. There is little accurate baseline information on clinical trial participation for patients with rare disease, use of centers of excellence for rare disease, or the survival benefit of clinical trial participation. Furthermore, comparisons about race and socioeconomic class to date have only looked at basic access to care and disease heterogeneity. Scientific evidence is needed to demonstrate improved outcomes and to develop standard practice guidelines for multidisciplinary models to better understand and improve care for these patients. There is no one-size-fits-all approach to building a sustainable multidisciplinary clinic. Ultimately, the best first step in planning for a multidisciplinary clinic is identification of and engagement with key stakeholders.

In Fig. 1, we have identified some of the key stakeholders for development of our multidisciplinary clinics at both the direct patient care and indirect support levels. Development of the CARE Clinic was supported by multiple individual departments (e.g., physical therapy and rehabilitation, nutrition, social work, and case management) as well as the institutional leadership. To garner institutional-level support, a comprehensive business plan was created to establish the need for such services and to define specific "asks" as well as plan for future growth and development. From a clinical perspective, the trained oncology-specific personnel were already on staff in multiple departments and provisions were made to support continuing education, particularly with a focus in gerontology to enhance clinical staff expertise. Educational opportunities may be available through affiliate colleges within larger universities, but we have also partnered with local aging resource agencies to learn about available supportive services to support community-dwelling older adults. Our CARE Clinic team members also participate in national and international groups such as the Cancer and Aging Research Group (CARG), the International Society for Geriatric Oncology (SIOG), and geriatric or aging-specific committees within the American Society of Hematology (ASH), American Society of Clinical Oncology (ASCO), and American Society for Transplantation and Cellular Therapy (ASTCT). 
Fig. 1 Key stakeholders in multidisciplinary clinic models

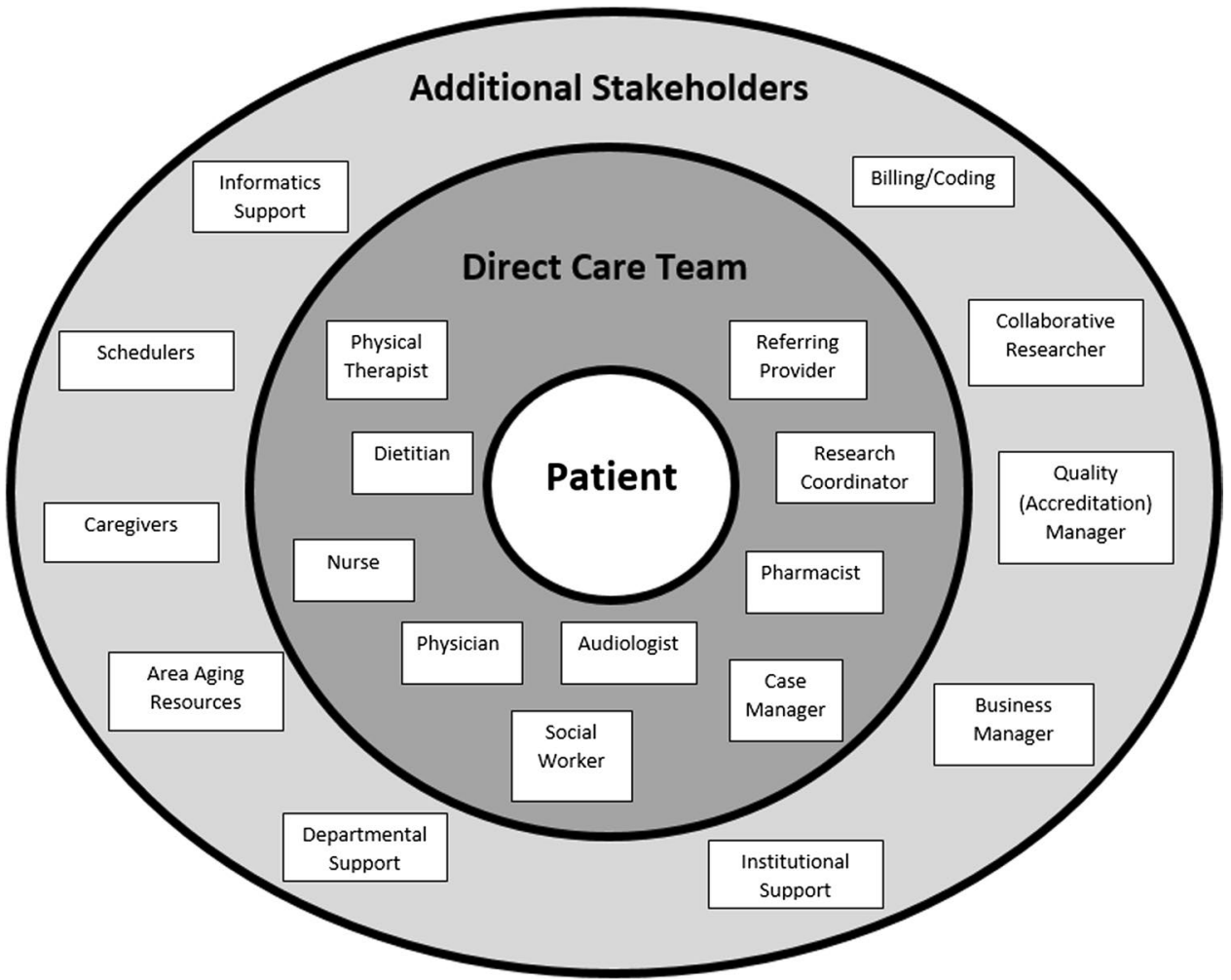

\section{Future Directions}

We plan to continue to grow our Oncogeriatrics Program through the established business plan but also through clinical care directed by our research efforts and monitored quality metrics. Our program benefits from having both a business manager and a quality manager who work together to advance the program. Our business manager oversees community outreach programs to facilitate referrals and, in the future, to develop partnerships with opportunity for extension into more rural areas. Our experience in converting the multidisciplinary clinic from in-person to telehealth during the COVID-19 pandemic temporarily allowed for greater accessibility to the clinic; however, we have seen lower utilization of the telehealth clinic option as patients have become more comfortable with returning to in-person visits. We are continuing to explore how to best utilize a telehealth clinic to afford greater accessibility. Our quality manager currently tracks wait time for appointments and referrals to CARE clinic by a provider as well as feedback through patient experience surveys. We have also retrospectively evaluated survival and hospitalization rates in patients seen in the CARE clinic and will soon open a prospective cohort study to be able to better track outcome measures of healthcare resource utilization and survival.

\section{Conclusion}

Older adults with hematologic malignancies are likely to increase as the population grows. These adults have increasing numbers of chronic co-morbidities and social and economic needs that must be balanced with emerging evidence, treatment tolerability, prognosis, and quality of life. The multidisciplinary oncology clinic offers a collaborative and integrative approach that balances these complexities while identifying and addressing geriatric syndromes and other needs. In this paper, we briefly describe the evolution of multidisciplinary care into the oncogeriatric population and offer an overview of our CAC and CARE Clinics. We are working to increase multidisciplinary care to those with hematologic malignancies and disorders. More research is needed to determine whether geriatric assessment and intervention tools should differ between hematologic malignancies and solid tumor as well as how to adapt the multidisciplinary model of care to meet these unique needs.

Author Contribution Manuscript preparation: SAW, ES, JV, NB. Manuscript editing: SAW, ES, JV, NB, AER, UB. All authors have reviewed and consent to submission of manuscript in its current form.

Data Availability Not applicable. 
Code Availability Not applicable.

\section{Declarations}

Ethics Approval Not applicable.

Consent to Participate Not applicable.

Consent for Publication Not applicable.

Human and Animal Rights and Informed Consent This article does not contain any studies with human or animal subjects performed by any of the authors.

Conflict of Interest The authors have no conflicts of interest relevant to the content of this manuscript.

\section{References}

1. Committe on the Science of Team Science, et al. Funding and evaluation of team science, in Enhacing and effectiveness of team science, Cooke N, Hilton M, Editors. 2015, National Academies Press: Washington (DC).

2. Choi BCK, Pak AWP. Multidisciplinarity, interdisciplinarity and transdisciplinarity in health research, services, education and policy: 1. Definitions, objectives, and evidence of effectiveness. Clinical and investigative medicine. Medecine clinique et experimentale. 2006;29(6):351-364.

3. Mion L, et al. Interdisciplinary care for older adults with complex needs: American Geriatrics Society position statement. J Am Geriatr Soc. 2006;54(5):849-52.

4. Counsell S. Outpatient care systems, in Geriatrics review syllabus: a core curriculum in geriatric medicine. D. A, S. G, Editors. 2013, American Geriatrics Society: New York. p. 189.

5. Campbell LJ, Cole KD. Geriatric assessment teams. Clin Geriatr Med. 1987;3(1):99-110.

6. Wieland $\mathrm{D}$, et al. The interdisciplinary team in geriatric care. American Behavioral Scientist. 1996;39(6):655-664.

7. Terret $\mathrm{C}$, et al. Multidisciplinary approach to the geriatric oncology patient. J Clin Oncol. 2007;25(14):1876-81.

8. Overcash J, et al. Geriatric oncology ambulatory care clinics. Oncology Issues. 2018;33(3):54-61.

9. The John A. Hartford Foundation. Tools you can use. A new appraoch to treating older adults. June 16, 2013 [cited 2021 March 12]; Available from: https://www.johnahartford.org/blog/ view/tools-you-can-use-a-new-approach-to-treating-older-patie nts/.

10. Bouzereau V, et al. Experience of multidisciplinary assessment of elderly patients with cancer in a French general hospital during 1 year: a new model care study. J Geriatr Oncol. 2013;4(4):394-401.

11. Horvath LE, et al. Multidisciplinary care in the oncology setting: historical perspective and data from lung and gynecology multidisciplinary clinics. J Oncol Pract. 2010;6(6):e21-6.

12. $\mathrm{Vu} \mathrm{JV}$ et al. Development and characteristics of a multidisciplinary colorectal cancer clinic. Am J Surg. 2021 Apr;221(4):826-831.

13. Houssami N, Sainsbury R. Breast cancer: multidisciplinary care and clinical outcomes. Eur J Cancer. 2006;42(15):2480-91.

14. Gabel M, Hilton NE, Nathanson SD. Multidisciplinary breast cancer clinics. Do they work? Cancer. 1997;79(12):2380-4

15. Chirgwin J, et al. Does multidisciplinary care enhance the management of advanced breast cancer?: Evaluation of advanced breast cancer multidisciplinary team meetings. J Oncol Pract. 2010;6(6):294-300.

16. Engel-Nitz NM, et al. Diagnostic testing managed by hematopathology specialty and other laboratories: costs and patient diagnostic outcomes. BMC Clin Pathol. 2014;14:17.

17. Taylor J, Xiao W, Abdel-Wahab O. Diagnosis and classification of hematologic malignancies on the basis of genetics. Blood. 2017;130(4):410-23.

18. Hussaini M. Biomarkers in hematological malignancies: a review of molecular testing in hematopathology. Cancer Control. 2015;22(2):158-66.

19. Goede V, Stauder R. Multidisciplinary care in the hematology clinic: implementation of geriatric oncology. J Geriatr Oncol. 2019;10(3):497-503.

20. Manitta VJ, Philip JA, Cole-Sinclair MF. Palliative care and the hemato-oncological patient: can we live together? A review of the literature. J Palliat Med. 2010;13(8):1021-5.

21. Rabinowitz B. Interdisciplinary breast cancer care: declaring and improving the standard. Oncology (Williston Park), 2004;18(10):1263-8; discussion 1268-70, 1275.

22. Fennell ML, et al. The organization of multidisciplinary care teams: modeling internal and external influences on cancer care quality. J Natl Cancer Inst Monogr. 2010;2010(40):72-80.

23. Berry MG, et al. An audit of patient acceptance of one-stop diagnosis for symptomatic breast disease. Eur J Surg Oncol. 1998;24(6):492-5.

24. Frost $\mathrm{MH}$, et al. A multidisciplinary healthcare delivery model for women with breast cancer: patient satisfaction and physical and psychosocial adjustment. Oncol Nurs Forum. 1999;26(10):1673-80.

25. Conron M, et al. Analysis of multidisciplinary lung cancer practice. Intern Med J. 2007;37(1):18-25.

26. Stone CJL, et al. Improving timeliness of oncology assessment and cancer treatment through implementation of a multidisciplinary lung cancer clinic. J Oncol Pract. 2019;15(2):e169-77.

27. Kozak VN, et al. Multidisciplinary clinics for colorectal cancer care reduces treatment time. Clin Colorectal Cancer. 2017;16(4):366-71.

28. Borneman T, et al. An interdisciplinary care approach for integration of palliative care in lung cancer. Clin Lung Cancer. 2008;9(6):352-60.

29. Chock MM, et al. Impact of a structured multidisciplinary intervention on quality of life of older adults with advanced cancer. Int Psychogeriatr. 2013;25(12):2077-86.

30. Penel N, et al. Factors favouring palliative treatment multidisciplinary decisions for newly diagnosed visceral and soft tissue sarcomas. Clin Oncol (R Coll Radiol). 2008;20(7):523-7.

31. Coory M, et al. Systematic review of multidisciplinary teams in the management of lung cancer. Lung Cancer. 2008;60(1):14-21.

32. Holden CA, et al. A systematic scoping review of determinants of multidisciplinary cancer team access and decision-making in the management of older patients diagnosed with colorectal cancer. J Geriatr Oncol. 2020;11(6):909-16.

33. Festen S, et al. The effect of treatment modifications by an onco-geriatric MDT on one-year mortality, days spent at home and postoperative complications. J Geriatr Oncol. 2021 Jun;12(5):779-785.

34. Lin RJ, et al. Impact of geriatric vulnerabilities on allogeneic hematopoietic cell transplantation outcomes in older patients with hematologic malignancies. Bone Marrow Transplant. 2020;55(1):157-64.

35. Derman BA, et al., Recommendations and outcomes from a geriatric assessment guided multidisciplinary clinic prior to autologous stem cell transplant in older patients. J Geriatr Oncol. 2021 May;12(4):585-591 
36. Derman BA, et al. Results from a multidisciplinary clinic guided by geriatric assessment before stem cell transplantation in older adults. Blood Adv. 2019;3(22):3488-98.

37. Tyler $\mathrm{KH}$, et al. The role of an integrated multidisciplinary clinic in the management of patients with cutaneous lymphoma. Front Oncol. 2015;5:136.

38. Bolton KL, et al. The clinical management of clonal hematopoiesis: creation of a clonal hematopoiesis clinic. Hematol Oncol Clin North Am. 2020;34(2):357-67.

39. World Health Organization. Ageing and health. 2/5/18 [cited 2021 3/30/21]; Available from: https://www.who.int/news-room/factsheets/detail/ageing-and-health.

40. SEER Cancer Statistics Review. 1975-2017 November 2019 SEER data submission, posted to SEER web site April 2020; Available from: https://seer.cancer.gov/csr/1975_2017/.

41. Eichhorst B, Hallek M, Goede V. New treatment approaches in CLL: challenges and opportunities in the elderly. J Geriatr Oncol. 2016;7(5):375-82.

42. Di M, Huntington SF, Olszewski AJ. Challenges and opportunities in the management of diffuse large B-cell lymphoma in older patients. Oncologist. 2021;26(2):120-32.

43. Sekeres MA, et al. American Society of Hematology 2020 guidelines for treating newly diagnosed acute myeloid leukemia in older adults. Blood Adv. 2020;4(15):3528-49.

44. Palumbo A, et al. Geriatric assessment predicts survival and toxicities in elderly myeloma patients: an International Myeloma Working Group report. Blood. 2015;125(13):2068-74.
45. Engelhardt M, et al. A concise revised Myeloma Comorbidity Index as a valid prognostic instrument in a large cohort of 801 multiple myeloma patients. Haematologica. 2017;102(5):910-21.

46. Merli F, et al. Simplified geriatric assessment in older patients with diffuse large B-cell lymphoma: the prospective elderly project of the Fondazione Italiana Linfomi. 0(0):JCO.20.02465.

47. Scheepers ERM, et al. Geriatric assessment in older patients with a hematologic malignancy: a systematic review. Haematologica. 2020;105(6):1484-93.

48. Klepin HD, et al. Individualizing treatment decisions for older adults with hematologic malignancies. Am Soc Clin Oncol Educ Book. 2013;208-19.

49. clinicaltrials.gov. Search: active interventional trials enrolling older adults ( $>65$ years) with cancer, excluded pharmacologic intervention. [cited 2021 April 5]; Available from: www.clinicaltr ials.gov.

50. Presley CJ, et al. Implementing a multidisciplinary approach for older adults with Cancer: geriatric oncology in practice. BMC Geriatr. 2020;20(1):231.

51. Wall SA, et al. Multidisciplinary telemedicine and the importance of being seen. J Geriatr Oncol. 2020;11(8):1349-51.

Publisher's Note Springer Nature remains neutral with regard to jurisdictional claims in published maps and institutional affiliations. 